\title{
Die OSZE in Zentralasien: Warum eine Debatte über Polizeihilfe nötig ist
}

\author{
Cornelius Friesendorf
}

\section{Zusammenfassung}

Dieser Beitrag ruft zu einer Debatte über polizeiliche Aktivitäten der OSZE in Zentralasien auf und untersucht drei Fragen: Kann die OSZE die demokratische Polizeiarbeit in Zentralasien unterstützen? Inwieweit kann die OSZE zur Verbesserung der menschlichen Sicherheit beitragen? Was sind die Risiken polizeilicher Ausbildungs- und Ausstattungshilfe? Eine wesentliche Herausforderung bei der Unterstützung demokratischer Polizeiarbeit sind Widerstände durch zentralasiatische Regierungen. Weitere Hindernisse sind Polizeiprogramme anderer internationaler Akteure und institutionelle Besonderheiten der OSZE wie kurze Haushaltszyklen. Dieser Beitrag beschreibt, wie Teilnehmerstaaten Chancen nutzen und Risiken begrenzen können.

\section{Schlagworte}

OSZE, Zentralasien, polizeibezogene Aktivitäten, demokratische Regierungsführung, Menschenrechte

Bitte zitieren als: Cornelius Friesendorf, Die OSZE in Zentralasien: Warum eine Debatte über Polizeihilfe nötig ist, OSCE Insights 1 (Baden-Baden: Nomos, 2021),

https://doi.org/10.5771/9783748911630-01

\section{Einleitung}

Die OSZE engagiert sich seit Jahrzehnten für demokratische Polizeiarbeit. ${ }^{1}$ Demokratische Polizeiarbeit erfordert Rechenschaftspflicht und Kontrolle: die Polizei muss dem Gesetz unterstellt sein und Institutionen wie das Parlament und die Medien müssen die Polizei kontrollieren können. Demokratische Polizeiar-

* Dr. habil. Cornelius Friesendorf Institut für Friedensforschung und Sicherheitspolitik an der Universität Hamburg (IFSH)

friesendorf@ifsh.de beit heißt auch, dass die Polizei die Menschenrechte schützt und Bürger unterstützt. ${ }^{2}$

In der Praxis fällt es der OSZE jedoch zunehmend schwer, demokratische Polizeiarbeit zu stärken. Kurz bevor er im Juli 2020 seinen Posten als OSZEGeneralsekretär verlor, erklärte Thomas Greminger, er sei „besorgt über die Abnahme der Initiativen zu Menschenrechten und zur Rechenschaftspflicht der Polizei".3 In Zentralasien steht die OSZE vor erheblichen Herausforderungen: Die Regierungen dort bemühen sich um Unterstützung bei der Strafverfolgung und begrüßen Initiativen, die die Polizei ser- 
viceorientierter machen, an einer demokratischen Kontrolle der Polizei besteht jedoch kaum Interesse.

Dieser Beitrag ruft zu einer Debatte über OSZE-Polizeihilfe in Zentralasien auf. Der erste Teil bietet einen Überblick über die polizeibezogenen Aktivitäten der OSZE; der zweite beschreibt Polizeiarbeit in Zentralasien. Anschließend werden folgende Fragen gestellt:

- Kann die OSZE demokratische Polizeiarbeit in Zentralasien unterstützen?

- Kann die OSZE menschliche Sicherheit stärken?

- Was sind die Risiken einer Unterstützung der Strafverfolgung?

Ein zentrales Hindernis für demokratische Polizeiarbeit in Zentralasien - und genereller für gutes Regieren im Sicherheitssektor (Security Sector Governance and Reform, SSG/R) - sind patronale Strukturen, die im Gegensatz zu liberalen Normen stehen. Weitere Hindernisse sind der russische Widerstand gegen Demokratisierung, chinesische und auch westliche Ausbildungs- und Ausrüstungsprogramme sowie die knappen Ressourcen und kurzen Planungszyklen der OSZE. Diese Faktoren erschweren es der OSZE ihre umfassende Sicherheitsagenda umzusetzen und machen internationale Unterstützung der Strafverfolgung für die lokale Bevölkerung riskant.

Der letzte Abschnitt enthält Empfehlungen, wie Teilnehmerstaaten Chancen nutzen und die Risiken von Polizeihilfe begrenzen können. Regierungen sollten OSZE-Polizeihilfe diskutieren; gesellschaftliche Gruppen in Zentralasien, die Reformen fordern, unterstützen; das
OSZE-Sekretariat, die Feldoperationen und die Institutionen unterstützen; die Beteiligung der OSZE an der Strafverfolgung einschränken; und auf eine systematische externe und unabhängige Evaluierung drängen.

\section{Die OSZE und die internationale Polizeiunterstützung}

Die OSZE ist ein Vorreiter bei der demokratischen Polizeiarbeit. Der Leitfaden für demokratische Polizeiarbeit von 2008 ist weltweit zu einem Referenzdokument für Polizeireform geworden. ${ }^{4}$ Zahlreiche andere Dokumente zeigen ebenfalls das Engagement der OSZE in dieser Frage. ${ }^{5}$ Eine Schlüsselvereinbarung aus dem Jahr 2012 besagt:

Die Aktivitäten der OSZE im polizeilichen Bereich folgen den in den Dokumenten der Vereinten $\mathrm{Na}$ tionen und der OSZE festgeschriebenen Normen, Prinzipien und Standards wie der Charta der Vereinten Nationen, den maßgeblichen UN-Übereinkommen betreffend polizeibezogene Aktivitäten, der Schlussakte von Helsinki, dem Kopenhagener Dokument und den verschiedenen OSZE-Beschlüssen zu polizeibezogenen Aktivitäten. Diese Dokumente unterstreichen unter anderem die Bedeutung der Rechtsstaatlichkeit, der Achtung der Menschenrechte und Grundfreiheiten, einschließlich Gender- und Minderheitenfragen, der Partnerschaft von Polizei und Öffentlichkeit, einer wirksamen 
und rechenschaftspflichtigen Strafrechtspflege... ${ }^{6}$

Polizeibezogene Aktivitäten sind insbesondere mit der ersten (politischmilitärischen) Dimension der OSZE verbunden. Sie sind jedoch auch für die zweite Dimension (z.B. Korruptionsbekämpfung) und die dritte (z.B. menschenrechtsorientierte Polizeiarbeit) relevant. Während der Ständige Rat das wichtigste Beschlussfassungsorgan der OSZE ist, werden Aktivitäten von den Exekutivstrukturen der OSZE durchgeführt. Hierzu zählen die thematischen Einheiten des Sekretariats, insbesondere das Referat Strategische Polizeiangelegenheiten (Strategic Police Matters Unit, SPMU) und das Referat Grenzsicherung und Grenzmanagement (Border Security and Management Unit, BSMU), sowie die Feldoperationen. Auch die OSZE-Institutionen sind beteiligt. So fordert das Büro für demokratische Institutionen und Menschenrechte (ODIHR) die Einhaltung von Menschenrechtsstandards, der Hohe Kommissar für Nationale Minderheiten (HCNM) unterstützt die multiethnische Polizeiarbeit und der OSZEBeauftragte für Medienfreiheit (RFOM) fördert die freie Berichterstattung über Sicherheitsfragen.

Erste OSZE-Initiativen zur demokratischen Polizeiarbeit gab es auf dem Balkan. Dort hat die OSZE seit Anfang der 2000er Jahre maßgeblich zur Demokratisierung der Polizei beigetragen und dabei von umfassenden Mandaten und der Konditionalität der EU-Mitgliedschaft profitiert. ${ }^{7}$
Die OSZE teilt ihre polizeibezogenen Aktivitäten in zwei Bereiche auf: „allgemeine Entwicklung und Reform der Polizei“ und „Bedrohungen durch kriminelle Aktivitäten". ${ }^{8}$ Diese Kategorien sagen aber nichts über Unterstützung für demokratische Polizeiarbeit aus. Dieser Beitrag unterscheidet zwischen Aktivitäten, die

- demokratische Polizeiarbeit (Typ 1), - einen besseren Schutz der Menschenrechte durch die Polizei (Typ 2) und

- stärkere Strafverfolgung (Typ 3) betonen.

Während die externe Kontrolle über die Polizei etwa durch das Parlament oder die Zivilgesellschaft bei Typ 1 im Mittelpunkt steht, ist sie bei Typ 2 weniger institutionalisiert und spielt bei Typ 3 kaum eine Rolle. Eine externe Kontrolle ist wichtig, weil die interne Aufsicht durch Polizeivorgesetzte und die Regierung oft nicht ausreicht.

Internationale Akteure können die demokratische Polizeiarbeit (Typ 1) fördern, indem sie Änderungen des Rechtsrahmens unterstützen. Weniger ambitioniert können internationale Akteure Parlamentarier oder Journalisten über Möglichkeiten informieren, ihre Rechte auszuüben, oder Foren für Diskussionen zwischen Staat und Gesellschaft über Polizeireformen schaffen.

Internationale Akteure können auch den Schutz der menschlichen Sicherheit durch die Polizei verbessern (Typ 2). Ziel ist, Polizeigewalt und Korruption zu verringern, beispielsweise durch Anti-Folter-Training, und die Polizei für Probleme wie häusliche Gewalt oder Menschenhandel zu sensibilisieren. Aktivitäten des 
Typs 2 fördern die Interaktion zwischen der Polizei und der Öffentlichkeit, insbesondere durch bürgernahe Polizeiarbeit.

Internationale Akteure können weiterhin die Strafverfolgung durch Ausbildung und materielle Hilfe stärken (Typ 3). Hier sind transnationale Interaktionen von Polizisten wichtiger als die demokratische Kontrolle über die Polizei. Die Öffentlichkeit kann von technischer Polizeihilfe profitieren, falls die Stärkung des Staates zu mehr Schutz vor Kriminalität und Gewalt durch Dritte führt.

Jede dieser drei Typen hat Vorteile, aber auch Grenzen und Risiken. Die Vorund Nachteile spezifischer Ansätze hängen wesentlich vom lokalen Kontext ab. Zentralasien stellt in dieser Hinsicht besondere Herausforderungen dar.

\section{Polizeiarbeit in Zentralasien}

Die Frage, was demokratische Polizeiarbeit ausmacht, ist umstritten, weil Prinzipien wie Rechenschaftspflicht interpretationsbedürftig sind und selbst in liberalen Demokratien unterschiedlich ausgelegt werden. Darüber hinaus ist polizeiliches Fehlverhalten im gesamten OSZEGebiet ein Problem (wie sich durch die „Black Lives Matter"-Proteste im Sommer 2020 gezeigt hat). Dennoch ist demokratische Polizeiarbeit in Demokratien wahrscheinlicher als in Autokratien.

Die zentralasiatischen Staaten rangieren auf den Indizes für Menschenrechte und Demokratie weit unten. Freedom House stuft Turkmenistan, Tadschikistan und Usbekistan als „nicht frei“ ein und zählt sie zu den am wenigsten freien Staa- ten der Welt; Kasachstan ist auch „nicht frei“" und Kirgisistan nur "teilweise frei“.? Ungeachtet der methodischen Herausforderungen und normativen Annahmen solcher Rankings helfen sie, den nichtdemokratischen Charakter der Polizeiarbeit in Zentralasien zu verstehen, der von internationalen Organisationen, NGOs und Medien umfassend dokumentiert worden ist. ${ }^{10}$

Es gibt verschiedene Ursachen für polizeiliches Fehlverhalten. Einige Praktiken aus der Sowjetzeit bestehen nach wie vor. So muss die Polizei unrealistische Quoten der Verbrechensaufklärung erfüllen, was zum Erzwingen von Geständnissen anreizt. Niedrige Löhne, ein Mangel an Ausrüstung und wenig familienfreundliche Arbeitszeiten sind weitere Faktoren. ${ }^{11}$ Auch politischer Druck spielt eine Rolle: Regierungen nutzen die Polizei als Instrument gegen die politische Opposition. Weiterhin tolerieren Politiker*innen polizeiliche Korruption im Gegenzug für politische Loyalität.

Problematisch sind auch die sich überschneidenden Befugnisse der Sicherheitsbehörden, wie die Verbreitung von Spezialeinheiten zeigt. In Kirgisistan verfügen das Innenministerium, aber auch der Sicherheitsdienst, die Nationalgarde und der Grenzschutz über Spezialeinheiten. ${ }^{12}$ In Usbekistan wurden der Nationalgarde Aufgaben bei der öffentlichen Ordnung und Terrorismusbekämpfung übertragen. ${ }^{13}$

Was die Kontrolle der Polizei angeht: Selbst wenn formale Strukturen vorhanden sind, werden sie in der Praxis durch informelle Regeln, die Präsidialverwaltungen, nationale Sicherheitsräte 
und Strafverfolgungsbehörden privilegieren, umgangen. Zentralasiatische Parlamentarier*innen zeigen wenig Neigung, die Exekutive herauszufordern. In Kirgisistan in den späten 2010er Jahren war die Aufsicht durch die Zhogorku Kenesh „unvollständig und inkonsistent, die parlamentarische Kontrolle über Sicherheitsund Strafverfolgungsbehörden findet sogar noch begrenzter und unregelmäßiger statt." ${ }^{14}$ Abgeordnete konzentrierten sich eher auf polizeiliches Fehlverhalten auf niedriger Ebene. ${ }^{15}$ Auch in Usbekistan hat das Parlament die Polizei kaum kontrolliert, und Menschenrechtsaktivist*innen fanden es schwierig, mit Abgeordneten zusammenzuarbeiten. ${ }^{16}$ Das tadschikische Parlament hat die Exekutivbeschlüsse weitgehend abgesegnet und die Abgeordneten zeigten wenig Neigung über Polizeigewalt zu diskutieren, möglicherweise, weil viele von ihnen Verbindungen zu den Sicherheitskräften hatten. ${ }^{17}$

Auch die Kontrolle durch Ombudsinstitutionen war begrenzt. Das kirgisische Ombudsbüro verfügt über erhebliche Befugnisse und Ressourcen und untersuchte in den 2010er Jahren eine Vielzahl von Beschwerden. Aber es gab Zweifel an der Unabhängigkeit des Bürgerbeauftragten, nicht zuletzt wegen seiner früheren Karriere in den Nachrichtendiensten; außerdem sagten Beobachter*innen, dass das Parlament seine Empfehlungen häufig ignorierte. ${ }^{18}$ Darüber hinaus haben die usbekischen und tadschikischen Ombudsleute laut Kritiker*innen Beschwerden gegen die Regierung nicht ordnungsgemäß untersucht und im Falle Tadschi- kistans Menschenrechtsverletzungen geleugnet. ${ }^{19}$

Diese Bedingungen schaffen Grenzen und Risiken für polizeiliche Aktivitäten der OSZE. In den folgenden Abschnitten werden drei Fragen aufgeworfen, die einer Debatte bedürfen.

\section{Kann die OSZE die demokratische Polizeiarbeit in Zentralasien unterstützen?}

Die Unterstützung der demokratischen Polizeiarbeit (Typ 1) zielt darauf ab, die Interaktion staatlicher Institutionen untereinander und mit der Gesellschaft zu verändern. Weil sie so anspruchsvoll ist, könnte die Aufgabe für die OSZE in Zentralasien zu groß sein.

In Kirgistan beendete die Regierung 2016 die Community Security Initiative (CSI). Diese Initiative, an der OSZE-Polizeiberater*innen beteiligt waren, sollte Vertrauen zwischen der Polizei und der Öffentlichkeit sowie - nach der interethnischen Gewalt im Jahr 2010 - zwischen ethnischen Kirgisen und Usbeken im Süden aufbauen. In der Anfangsphase der CSI ermöglichte die Polizei auch Gespräche zwischen der Regierung und Polizeireformexperten*innen aus der Zivilgesellschaft. ${ }^{20}$

Die nachfolgenden Aktivitäten waren weniger ambitioniert. Das Programmbüro in Bischkek (POiB) förderte weiterhin Diskussionen in Räten, die Staat und Zivilgesellschaft zusammenbrachten, aber diese Diskussionen führten zu eher moderaten Forderungen an das Innenministerium. Bezeichnenderweise war der 
Hauptpartner des POiB für Diskussionen zum Thema Kontrolle ausgerechnet dieses Ministerium, wobei das POiB erklärte, dass es „das Innenministerium bei der Förderung der parlamentarischen und zivilen Kontrolle der Strafverfolgungsreform unterstützte“. ${ }^{21}$ Zwar thematisierte das POiB bei Projekten mit den Verwaltungsakademien zentralasiatischer Staaten Fragen von SSG/R. Für einige kirgisische Polizeiexperten arbeitete die OSZE insgesamt aber zu wenig mit regierungskritischen Gruppen zusammen, und sie schätzten die Polizeireform als oberflächlich ein. ${ }^{22}$

In Usbekistan bemühte sich Präsident Shavkat Mirziyoyev um eine aktivere Rolle des Parlaments, z.B. bei der Kontrolle des Haushaltes. ${ }^{23}$ Dies eröffnete Möglichkeiten für ODIHR und den Projektkoordinator in Usbekistan (PCUz). Sie organisierten unter anderem eine Konferenz zur „Demokratisierung der Gesetzgebung und der Strafverfolgungspraxis" in Usbekistan. ${ }^{24}$ Aber Wünsche nach technischer Polizeihilfe überwogen das Interesse an Unterstützung bei der guten Regierungsführung. ${ }^{25}$

\section{Die Grenzen der OSZE verstehen}

Die OSZE ist zwar mehr als ein zwischenstaatliches Forum oder ein Instrument von Staaten, sie ist jedoch eine auf Konsens zwischen Regierungen beruhende Organisation, und eine demokratische Polizeiarbeit steht tendenziell im Widerspruch zu den Interessen der zentralasiatischen Regierungen.
Widerstand gegen demokratische Reformen ist mit innenpolitischen Logiken verbunden. Henry Hale zeigt, wie patronale Herrschaft, die auf personalisierten Netzwerken basiert, die postsowjetischen Regime dominiert, in der Regel mit Präsidenten als Mittelpunkt, die Personen belohnen oder bestrafen können. ${ }^{26}$ Die Mitglieder dominanter Netzwerke bekleiden offizielle Positionen, aber diese Netzwerke reichen auch in nichtstaatliche Sektoren hinein. Die Kontrolle durch staatliche Institutionen wie das Parlament ist oft ineffektiv, weil Eliten ihre Patrone nicht herausfordern, solange sie diese als stark betrachten. Informelle Normen, die sich um persönliche Bekanntschaften drehen, sind wichtiger als abstrakte Prinzipien wie Rechtsstaatlichkeit. Politischer Wandel, selbst der gewaltsame Sturz von Regierungen, darf nicht mit Demokratisierung verwechselt werden; vielmehr bedeutet eine solche Dynamik, dass wechselnde Erwartungen der Elite neue Patrone an die Macht bringen. Internationalen Akteuren fehlen Hale zufolge die Druckmittel und die Verbindungen, um die klientelistische Politik im postsowjetischen Raum zu verändern. ${ }^{27}$

Für Liberale ist diese Analyse ernüchternd. Westliche Regierungen können versuchen, demokratische Regierungsführung zu unterstützen und außerbudgetäre Mittel, die keinen Konsens zwischen Teilnehmerstaaten erfordern, dafür bereitzustellen. Allerdings würde die Einführung einer wirksamen Kontrolle über die Exekutive für die herrschenden Netzwerke eine Gefahr darstellen. Eine demokratische Polizeiführung wür- 
de darüber hinaus eine Stärkung formaler gegenüber informellen Normen erfordern, was bestenfalls ein langsamer Prozess ist.

Bereits 2005 stellte eine Evaluierung fest, dass das „Klima für SSR in Zentralasien ungünstig ist, sowohl infolge des globalen ,Krieges gegen den Terror ${ }^{6}$ als auch aufgrund der Art der politischen Regime, die in der gesamten Region vorherrschen. Legislative und Justiz sind schwach, die Medien kraftlos und die Zivilgesellschaft nur wenig aktiv; all das hat die konservative Einstellung der zentralasiatischen Regime noch verstärkt. “28 Fünfzehn Jahre später gab es zwar ein stärkeres rhetorisches Bekenntnis zur Reform. Dennoch hat die Polizeiarbeit keine großen Fortschritte gemacht, was zeigt, wie widerstandsfähig Patronalismus ist.

\section{Wie Staaten die OSZE kontrollieren}

Die zentralasiatischen Staaten haben verschiedene Möglichkeiten, OSZE-Aktivitäten zu verhindern. Vor allem sind sie als gleichberechtigte Mitglieder der Organisation nicht nur Gastgeber von Feldoperationen, sondern entscheiden mit über Mandate.

Die Mandate von Feldoperationen sind immer restriktiver geworden, was sich in Namensänderungen widerspiegelt: Aus dem OSZE-Zentrum in Astana wurde 2015 das Programmbüro in Astana. Das OSZE-Zentrum in Usbekistan wurde 2006 in das Büro des OSZEProjektkoordinators umgewandelt. Das OSZE-Zentrum in Bischkek wurde 2016 in das OSZE-Programmbüro umbenannt und die Außenstelle in Osh wurde geschlossen. Das OSZE-Programmbüro in Duschanbe ersetzte 2017 das OSZE-Büro. Turkmenistan hat immer noch das OSZE-Zentrum in Aschgabat, das jedoch nur einen begrenzten Aufgabenbereich hat.

Zentralasiatische Regierungen kontrollieren die OSZE auch bei der Implementierung. Aktivitäten bedürfen ihrer Genehmigung, oft bis hin zu konkreten Projekten. Regierungen geben auch interpretative Erklärungen ab, die die Strukturen der OSZE dazu zwingen, nur solche Aktivitäten durchzuführen, die ausdrücklich durch Mandate abgedeckt sind. Auch Russland gibt solche Erklärungen ab. ${ }^{29}$

Regierungspräferenzen wirken auch indirekt: OSZE-Exekutivstrukturen werden risikoscheu. Internationale Mitarbeiter*innen im Wiener Sekretariat und vor Ort ahnen voraus, welche Aktivitäten zentralasiatische Regierungen billigen werden und neigen dazu, auf Nummer sicher zu gehen. Lokale Mitarbeiter*innen haben besonderen Grund vorsichtig zu sein, da der schwache Rechtsstatus der OSZE es der Organisation erschwert, ihre Fürsorgepflicht auszuüben. ${ }^{30}$ Auch haben sie wenig Chancen, innerhalb der OSZE (die keine Karriereorganisation ist) voranzukommen, und tendieren dazu, den $\mathrm{Ar}$ beitsmarkt, einschließlich staatlicher Arbeitsplätze, im Auge zu behalten. Das mindert ihre Neigung, sich für Demokratisierung einzusetzen.

Ein weiteres institutionelles Hindernis für die Verfolgung langfristiger Ziele ist die Finanzierung. Der OSZE-Haushaltszyklus beträgt in der Regel ein 
Jahr, und ein Großteil der Mittel wird durch außerbudgetäre Projekte bereitgestellt und fließt im Laufe des Jahres ein. Das steht einer strategischen Planung und der Verfolgung ehrgeiziger Ziele wie demokratische Regierungsführung entgegen. Eine 2013 veröffentlichte Bewertung der SSG/R-Aktivitäten der OSZE trifft auch auf die polizeibezogenen Aktivitäten der Organisation in Zentralasien zu:

Die Projekte sind oft ad hoc, basieren auf Anfragen der Teilnehmerstaaten und unmittelbar verfügbarem Fachwissen, und werden von den Prioritäten der einzelnen Staaten, die außerbudgetäre Mittel und sekundiertes Personal stellen, bestimmt. Infolgedessen werden Projekte häufig nicht in eine „Kette“ von Aktivitäten, die ein breites Ziel verfolgen, eingebunden, was ihre Wirksamkeit erheblich verringert. ${ }^{31}$

Unsicherheiten bezüglich der Verlängerung des Mandats, die Gefahr, dass die Gastgeberstaaten selbst den in Memoranda of Understanding festgelegten Programmen nicht mehr zustimmen, und mögliche Finanzierungsengpässe schaffen Unsicherheit. Aus bürokratischer Sicht ist es daher rational kurzfristig zu planen.

\section{Kann die OSZE dazu beitragen die menschliche Sicherheit in Zentralasien zu schützen?}

Die menschliche Sicherheit - die Freiheit des Einzelnen von Angst und Not - fällt vor allem in die menschliche Dimension der OSZE. Aktivitäten des Typs
2, die sich mit Menschenhandel, Strafvollzugsreformen oder geschlechtsspezifischer Gewalt befassen, berühren weniger den Kern der Staatlichkeit als demokratische Regierungsführung und werden daher von autoritären Staaten toleriert oder sogar gefördert, da diese so Legitimität gewinnen können. Darüber hinaus können diese Aktivitäten flexibel an den lokalen Kontext angepasst werden, was sich gut mit dem Konsens innerhalb der SSG/R-Community gegen Standardlösungen vereinbaren lässt. Auch sind Aktivitäten des Typs 2 wie die bürgernahe Polizeiarbeit in der Regel inklusiv und passen somit gut zur historischen Rolle der OSZE, nicht gleichgesinnten Akteuren Diskussionsforen zu bieten.

Aus diesen Gründen haben Aktivitäten des Typs 2 in den 2010er Jahren einen großen Teil der polizeilichen Aktivitäten der OSZE in Zentralasien ausgemacht und wurden sorgfältig auf die Agenden der Regierungen zugeschnitten. Dazu gehörten Konferenzen, Workshops, Schulungen, Handbücher, Aufklärungskampagnen und Studienreisen für Regierungsvertreter. ${ }^{32}$

In Kirgisistan unterstützte die OSZE nach dem Ende des CSI weiterhin die bürgernahe Polizeiarbeit durch die Finanzierung von Minibussen, die die Interaktion zwischen der Polizei und der Öffentlichkeit in abgelegenen Gebieten erleichtern sollten, sowie durch die Unterstützung lokaler Gemeinderäte, die an der Steuerung dieses Programms beteiligt waren. Das Projektbüro in Bischkek half auch bei der Verbesserung der Verkehrssicherheit, bei der Schulung zu neuen Strafrechtsgesetzen, bei der Folterpräven- 
tion und dem Schutz von Opfern des Menschenhandels sowie bei der Umsetzung eines Verhaltenskodex für die Polizei.

In Usbekistan hat die OSZE in den letzten Jahren die Bemühungen des Präsidenten unterstützt, die öffentliche Verwaltung zu verbessern und grobe Menschenrechtsverletzungen zu bekämpfen. Die Folterprävention ist somit ein wichtiger Bestandteil der Aufgaben des Projektkoordinators und anderer OSZE-Institutionen geworden. ${ }^{33}$ Weitere Aktivitäten betrafen den Menschenhandel, die Öffentlichkeitsarbeit der Polizei und die Bemühungen der Regierung, gewalttätigem Extremismus und Radikalisierung, die zum Terrorismus führen (VERLT), entgegenzuwirken.

Selbst in Tadschikistan konnte die OSZE Themen menschlicher Sicherheit auf der Tagesordnung halten, indem sie Aktivitäten etwa zu geschlechtsspezifischer Gewalt, Folterprävention und Jugendgerichtsbarkeit organisierte. Außerdem förderte sie die bürgernahe Polizeiarbeit durch die Finanzierung und Ausstattung von Modellpolizeistationen und die Schulung von Polizisten*innen bei der Beantwortung von Bürgeranfragen. Darüber hinaus unterstützte die OSZE Gemeinderäte, die Polizei- und Gemeindevertreter*innen zusammenbrachten.

Herausforderungen bei der Verbesserung der menschlichen Sicherheit

Pragmatische Projekte, die keine wesentlichen Veränderungen bei der Regie- rungsführung verlangen, können vielen Menschen helfen. So wandten sich laut der OSZE viele Bürger an die von der OSZE geförderten mobilen Polizeiteams. Unklar ist allerdings auch hier, ob zugrundeliegende Annahmen stimmen.

Erstens scheint die OSZE davon auszugehen, die Zivilgesellschaft könne gestärkt und damit das Verhalten der Polizei verändert werden. Die Unterstützung der Zivilgesellschaft steht aber unter anderem vor dem Problem von Machtasymmetrien. Zentralasiatische Gemeinderäte, die als Diskussionsplattformen für die Polizeireform dienen, werden in der Regel vom Innenministerium und Sicherheitsdienst dominiert. In Tadschikistan nannte eine Evaluierung diese Räte ,regierungseigen" ${ }^{34}$ Weiterhin ist fraglich, wie inklusiv zivilgesellschaftliche Gruppen sind, und lokale Würdenträger sind nicht unbedingt Verfechter liberaler Normen, etwa bezüglich häuslicher Gewalt. Der Begriff „Zivilgesellschaft“ impliziert auch eine Unterscheidung zwischen Staat und Gesellschaft, die in patronalen Systemen stark verschwimmt.

OSZE-Polizeiprogramme basieren weiterhin auf der Annahme, dass Wertewandel möglich ist; tatsächlich hat das Streben nach einem Wertewandel eine lange Geschichte in der KSZE/OSZE. ${ }^{35}$ Die Hoffnung ist, dass die Polizei durch die Interaktion mit der Zivilgesellschaft und mit internationalen Experten*innen zu normkonformem Verhalten hin sozialisiert werden kann. Die Organisationstheorie zeigt aber, dass sich Organisationskulturen und -routinen nur langsam ändern, insbesondere in Sicherheitsbehörden, deren Mitglieder*innen orga- 
nisatorische Normen in jungen Jahren internalisieren und die durch Hierarchie und Gruppenzwang geprägt sind. ${ }^{36} \mathrm{Da}$ rüber hinaus prägt die Politik das Verhalten von Sicherheitsbehörden. Korruption auf Regierungsebene sendet ein Signal aus, dass Korruption auf der Straße toleriert wird. Auch tendieren patronale Netzwerke dazu, diejenigen, die tiefgreifende Reformen fordern, unter Druck zu setzen.

Eine dritte Annahme scheint den Bemühungen der OSZE zugrunde zu liegen: dass kleine Schritte ihre eigene Dynamik entwickeln werden. So mögen Polizeireformer darauf hoffen, dass sich Modell-Polizeistationen im Land vervielfachen. Die Regierung kann bürgernahe Polizeiarbeit jedoch beenden oder verwässern, wie im Falle der Community Security Initiative in Kirgisistan. Polizeireformen können über Jahrzehnte andauern, ohne die Polizei zu demokratisieren. Zentralasiatische Staaten sind geschickt darin, Reformen zu beschleunigen oder $\mathrm{zu}$ verlangsamen und deren Inhalt $\mathrm{zu}$ kontrollieren.

Aktivitäten des Typs 2 riskieren daher, autoritäre Modernisierung zu stützen, also die Bemühungen illiberaler Staaten, Legitimität zu erlangen, ohne die klientelistische Regierungsführung zu ändern. Dieses Risiko muss gegen jede Verbesserung der menschlichen Sicherheit abgewogen werden. Es ist beim dritten Typ der Polizeihilfe noch höher.

\section{Wie risikoreich ist technische Polizeihilfe?}

Strafverfolgungshilfe kann die Fähigkeit der Polizei verbessern, Verbrechen zu verhindern und aufzuklären. Sie kann auch das polizeiliche Vorgehen mit den Menschenrechten in Einklang bringen, etwa wenn die Polizei in die Lage versetzt wird, sich auf forensische Beweise zu stützen anstatt auf erzwungene Geständnisse.

Aktivitäten des Typs 3 sind von zentraler Bedeutung für die Arbeit der OSZE in Zentralasien. Die Jahresberichte des Generalsekretärs über polizeibezogene Aktivitäten enthalten umfangreiche Auflistungen über Zusammenarbeit in Bereichen wie organisierte Kriminalität, Ermittlungen und Analysen, grenzübergreifende Zusammenarbeit, Terrorismusfinanzierung und VERLT, illegale Drogen und chemische Grundstoffe, Bekämpfung der Geldwäsche, Beschlagnahmung, Menschenhandel, migrationsbezogene Kriminalität, Grenzsicherheit und Cyberkriminalität. Die OSZE hat vor allem Ausbildungs- und Ausstattungshilfe geleistet. Darüber hinaus dienen internationale Studienreisen dem Austausch bewährter Verfahren (best practices) und dem Aufbau transnationaler polizeilicher Netzwerke.

Aktivitäten des Typs 3 sind aus mehreren Gründen beliebt. Am wichtigsten ist, dass die zentralasiatischen Regierungen solche Programme und Projekte anfragen. In der Tat: Regierungen beklagen sich mitunter sogar darüber, dass die OSZE nicht genug davon liefert. ${ }^{37}$ Institutionelle Kulturen und Anreize innerhalb der OSZE spielen ebenfalls eine 
Schlüsselrolle. Die für die Umsetzung der OSZE-Politik verantwortlichen Personen, wie etwa SPMU-Mitarbeiter*innen, kommen häufig aus den Sicherheitskräften und sind daher geneigt, sich für die Verbesserung der Polizeikapazitäten einzusetzen. Die Unterstützung der Strafverfolgung ermöglicht es dem Sekretariat und den Feldoperationen, über Aktivitäten zu berichten, Budgets zu sichern und Mittel schnell auszugeben. Polizeihilfe unterstützt daher eine Hypothese aus der Wissenschaft, dass nicht die OSZE die zentralasiatischen Staaten beeinflusst, sondern diese Staaten die OSZE als Instrument zur Erhaltung des Status quo nutzen. ${ }^{38}$

Ein weiterer Grund für die Dominanz von technischer Polizeihilfe ist, dass andere internationale Akteure solche Hilfe leisten, was die OSZE unter Druck setzt dies ebenfalls zu tun, um von den zentralasiatischen Staaten als relevant angesehen zu werden. Beispiele sind die Rolle der Europäischen Union (EU) beim Grenzmanagement, die Polizeiprogramme des Büros der Vereinten Nationen für Drogen und Verbrechensbekämpfung (UNODC) und bilaterale Hilfe der USA. Viele Geberprogramme externalisieren eigene innenpolitische Besorgnisse über Drogenhandel, Terrorismus oder illegale Migration und stärken so unbeabsichtigt zentralasiatische Machtministerien. ${ }^{39}$ Russland arbeitet ebenfalls eng mit den zentralasiatischen Sicherheitskräften zusammen, während China zunehmend seine eigenen autoritären Polizeipraktiken exportiert.

\section{Unterstützung der Strafverfolgung als Einfallstor für Reformen?}

Die OSZE integriert (im Einklang mit ihrer umfassenden Sicherheitsagenda) Menschenrechtselemente in ihre technische Polizeihilfe. Tatsächlich kann technische Polizeihilfe Menschenrechte stärken, beispielsweise durch Diskussionen und Praxisunterricht zu Verpflichtungen, Verdächtigte nicht zu misshandeln und zu foltern.

Nachhaltigen Veränderungen stehen aber die oben erwähnten organisatorischen Beharrungskräfte und die Risikoaversion von OSZE-Mitarbeiter*innen entgegen. Darüber hinaus ist die Ausbildungshilfe nicht nachhaltig, wenn neue Fähigkeiten nicht routinemäßig angewendet werden. In Zentralasien spricht das inländische institutionelle Umfeld gegen die Internalisierung von menschenrechtlichen Ausbildungsinhalten. Was Materialhilfe angeht, so rät David Bayley internationalen Akteuren, diese "nur $\mathrm{zu}$ leisten, um die Kosten für Reformen, die lokal akzeptiert sind, zu decken“. Die Hoffnung, durch Materialhilfe Widerstände gegen Reformen zu schwächen, sei unrealistisch. ${ }^{40}$

Aktivitäten vom Typ 3 können für die Förderung demokratischer Polizeiarbeit nicht nur ineffektiv sein, sie bergen unter autoritären Bedingungen auch erhebliche Risiken. Kritiker*innen haben auf die Gefahr hingewiesen, dass OSZE-Polizeihilfe die Repression unbeabsichtigt verstärken kann. ${ }^{41}$ Hinzu kommen weitere, weniger sichtbare Risiken, insbesondere die Stärkung der Legitimität autoritärer Staaten. Zentralasiatische Regierungen bekennen 
sich rhetorisch zur demokratischen Polizeiarbeit. Aber die formale Institutionalisierung von Normen etwa zur Gleichstellung der Geschlechter ist ohne Zeitpläne und nachfolgende Kontrolle nicht dasselbe wie eine Umverteilung der Macht. Der tadschikische Innenminister hat zum Beispiel in Wien Anfang 2018

die Bereiche vorgestellt, in denen die weitere Unterstützung der OSZE-Teilnehmerstaaten vorrangig gebraucht würde, wobei er ausdrücklich Fragen wie neue Polizeiuniformen, die Beschaffung neuer Ausrüstung für die Polizei und die Automatisierung aller Kommunikationsprozesse in den Strukturen des Innenministeriums erwähnte. In der anschließenden Diskussion ging der Minister auf die Themen bürgernahe Polizeiarbeit und Gleichstellung ein [...]. ${ }^{42}$

\section{Schlussfolgerungen und Empfehlungen}

Es gibt kaum eine öffentliche Debatte über polizeibezogene Aktivitäten der OSZE in Zentralasien und darüber, ob zugrundeliegende Annahmen plausibel sind und auf wissenschaftlichen Erkenntnissen basieren. Teilnehmerstaaten, die über Effektivität und Risiken besorgt sind, sollten:

- Polizeibezogene Aktivitäten debattieren. Die Teilnehmerstaaten sollten im Ständigen Rat, im Sicherheitsausschuss und in der OSZE-Gruppe der Freunde der SSG/R die Möglichkeiten, Grenzen und Risiken polizeibezo- gener OSZE-Aktivitäten erörtern. Sie sollten unterstreichen, dass diese Aktivitäten dimensionenübergreifend und als solche fest in der menschlichen Dimension verankert sind. Weiterhin sollten westliche Staaten die Polizeiarbeit im eigenen Land verbessern, um Vorbilder für andere Länder zu schaffen. Die OSZE sollte dafür auch westlich von Wien Polizeireformen unterstützen.

- Konzepte und Erfolgsmeldungen hinterfragen. Staaten sollten Erfolgsmeldungen in OSZE-Berichten und Schlagworte wie demokratische Polizeiarbeit, SSG/R und Kapazitätsaufbau hinterfragen und prüfen, ob die OSZE-Rhetorik mit der Praxis übereinstimmt. Sie sollten betonen, dass Ausbildungs- und Materialhilfe ohne überprüfbare Schritte zur besseren Regierungsführung nicht mit dem Konzept von umfassender Sicherheit vereinbar ist.

- Reformer identifizieren und unterstützen. Demokratische Polizeiarbeit hängt von einer umfassenden SSG/RStrategie und von Demokratisierung $\mathrm{ab}$, die überall ein langfristiger Prozess ist. Dennoch kann die OSZE innerstaatliche Reformer unterstützen, insbesondere in kritischen Phasen, wenn die politischen Bedingungen dies zulassen (wie in Kirgisistan nach der Revolution von 2010). Die OSZE sollte die vielversprechendsten Change Agents identifizieren und anerkennen, dass deren Unterstützung ein politischer, kein technischer Prozess ist und als solcher Kompromisse erfordert. So kann es sein, dass zivilgesellschaftliche 
Gruppen, die die Werte der OSZE teilen, innenpolitisch kaum Einfluss haben, während Gruppen mit innenpolitischer Durchsetzungskraft Teil des Regimes sind. In Zentralasien gibt es sachkundige Befürworter der Polizeireform, die OSZE-Unterstützung brauchen, etwa durch Beratung beim Management und Lobbying. Weitere Ansätze sind die Schulung von Journalisten*innen sowie die Unterstützung von Forschungsarbeiten zu SSG/R an der OSZE-Akademie in Bischkek.

- Unterstützung der OSZE-Exekutivstrukturen und Institutionen. Das OSZE-Sekretariat, die Feldoperationen und die Institutionen benötigen finanzielle und personelle Unterstützung für Projekte des Typs 1 und 2, auch durch extrabudgetäre Mittel. Sie brauchen auch politischen Rückhalt, wie z.B. die Zusicherung, dass die OSZE-Mitarbeiter*innen, einschließlich der lokalen Bediensteten, nicht allein gelassen werden, wenn sie Gastgeberstaaten missfallen.

- Ausbildungs- und Ausstattungshilfe reduzieren. Die Risiken von Typ-3-Aktivitäten überwiegen die Vorteile für die zentralasiatische Bevölkerung. Eine verbesserte Polizeiarbeit oder den Schutz der menschlichen Sicherheit zur Bedingung von Ausbildungs- und Materialhilfe $\mathrm{zu}$ machen oder beim Training auch über Menschenrechte zu sprechen ist keine Lösung. Zentralasiatische Staaten errichten demokratische Fassaden und können Projekte mikromanagen, und das Konsensprinzip der OSZE lässt wenig Raum für
Konditionalität im Stil der EU. Der Abbau oder die Einstellung von Ausbildungs- und Ausrüstungsprogrammen steht im Einklang mit dem Dono-harm-Prinzip. Demokratische Staaten sollten auch ihre Strafverfolgungshilfe für Zentralasien durch andere internationale Organisationen und bilaterale Programme überdenken, um den Wettbewerbsdruck auf die OSZE zu verringern. Man könnte einwenden, dass dies transnationaler organisierter Kriminalität oder dem Terrorismus zugutekommt. Dieser Einwand unterschätzt jedoch die negativen Folgen einer prinzipienlosen Hilfe, nicht nur für die Menschen vor Ort, sondern auch für westliche Interessen. Schließlich schafft Repression einen fruchtbaren Boden für transnationale Sicherheitsrisiken.

Eine zentrale Voraussetzung für eine faktenbasierte Diskussion über OSZE-Polizeihilfe ist Evaluierung. Die Teilnehmerstaaten sollten Aktivitäten und zugrunde liegende Annahmen überprüfen und die internen Evaluierungen des Büros für Innenrevision (Office of Internal Oversight) systematisch erörtern. Die Selbstevaluierungen von Feldoperationen verdienen ebenfalls eine genauere Prüfung. Darüber hinaus könnte die OSZE von mehr externen unabhängigen Evaluierungen profitieren sowie von innovativen Methoden, wie sie beispielsweise von der Ethnographie angewendet werden. Dichte Beschreibungen oder Umfragen zum öffentlichen Vertrauen in die Polizei liefern oft bessere Einblicke als bürokratische Praktiken aus dem New Public Management. 
Die Teilnehmerstaaten sollten auch darüber diskutieren, wie Informationen veröffentlicht werden können, um eine offene und informierte Debatte zu ermöglichen. Die OSZE produziert große Mengen an Informationen, aber öffentlich zugängliche Dokumente lassen viele Fragen offen. Die Öffentlichkeit weiß wenig über Theorien des Wandels, ihre Prämissen oder darüber, welche Staaten wie viele außerbudgetäre Mittel für welche Projekte bereitstellen. Abhilfe könnten Aufsichtsgremien für bestimmte Projekte und Programme schaffen, die ihre Ergebnisse zumindest in Teilen veröffentlichen.

Idealerweise sollten Evaluationen zeigen, ob polizeibezogene Aktivitäten der OSZE das Verhalten der Polizei (outcome) und die Kriminalität und Gewalt durch Dritte (impact) verändert haben. Hierfür nötige Daten sind aufgrund von Kontextfaktoren wie wirtschaftlichen oder demografischen Veränderungen oder der Polizeihilfe anderer internationaler Akteure allerdings schwer zu erheben. Hinzu kommt die Schwierigkeit, von zentralasiatischen Regierungen verlässliche Daten zu erhalten.

Auch bessere Evaluierungen werden daher Fragen offen lassen. Trotzdem sollte OSZE-Polizeihilfe genauer überprüft werden. Es reicht nicht, zu glauben, dass die OSZE komplexe Probleme in einem schwierigen politischen Umfeld mit einem kleinen Haushalt zwar nicht lösen kann, die Polizei in Zentralasien ohne die OSZE aber noch problematischer wäre. Das mag wahr sein, aber auf der Grundlage ungeprüfter Annahmen lässt sich selten gute Politik machen.

\section{Notizen}

1 Mein Dank geht an die Interviewpartner für Informationen und an meine Kolleg*innen am IFSH und die externen Gutachter*innen für Kommentare zu einer früheren Fassung.

Siehe David Bayley, Changing the Guard: Developing Democratic Police Abroad, Oxford: Oxford University Press, 2006, 18-22.

OSCE, Annual Report of the Secretary General on Police-Related Activities 2019, 2 July 2020, S. 2, https://www.osce. org/files/f/documents/2/6/456052.pdf.

OSCE, Guidebook on Democratic Policing by the Senior Police Advisor to the OSCE Secretary General, 24 January 2007, https://www.osce.org/secretariat/23 804.

Ein Überblick findet sich in: OSCE, Annual Report of the Secretary General on Police-Related Activities 2018, 8 August 2019, 157-162, https://www.osce.org/secr etariat/427427.

OSZE, Beschluss Nr. 1049: Strategischer Rahmen der OSZE für Aktivitäten im polizeilichen Bereich, PC.DEC/1049, 26. Juli 2012, S. 3, https://www.osce.org/f iles/f/documents/2/8/94941.pdf.

7 Steffen Eckhard, International Assistance to Police Reform: Managing Peacebuilding, Basingstoke: Palgrave Macmillan, 2016, Kapitel 3.

OSCE, Annual Reports of the Secretary General on Police-Related Activities, https://www.osce.org/spmu/108393.

9 Freedom House, Countries and Territories, Global Freedom Scores, https:// freedomhouse.org/countries/freedom-wo $\mathrm{rld} /$ scores? sort=asc\&order=Total\%20Sco re $\% 20$ and $\% 20$ Status, accessed on 5 June 2020.

10 Siehe beispielsweise International Partnership for Human Rights: Torture in Central Asia: Time to Break the Vicious 
Circle, 26 June 2019, https://www.iphron line.org/torture-in-central-asia-time-to-bre ak-the-vicious-circle.html.

11 Siehe International Crisis Group, Central Asia: The Politics of Police Reform, 10 December 2002, https://d2071andvip0wj. cloudfront.net/42-central-asia-the-politics -of-police-reform.pdf.

12 Interview mit einem Sicherheitsexperten, Bischkek, 7. März 2019.

13 Umida Hashimova, ,The National Guard of Uzbekistan: Rising Profile, Lingering Problems“, The Diplomat, 22 February 2019, https://thediplomat.com/2019/02/ the-national-guard-of-uzbekistan-rising-pr ofile-lingering-problems/.

14 Aida Alymbaeva/Rustam Burnashev/ Grazvydas Jasutis/Parviz Mullojanov/ Richard Steyne/Farkhod Tolipov, Parliamentary Oversight of the Security Sector: Case Studies from Central Asia, Geneva Centre for Security Sector Governance (DCAF), 2020, S. 28.

15 Interview mit einer kirgisischen Nichtregierungsorganisation, Bischkek, 11. März 2019.

16 Interviews mit einem NGO-Mitglied und einer Menschenrechtsgruppe, Taschkent, Juni 2019.

17 Siehe Alymbaeva et al., a.a.O. (Anm. 14), S. 47-54.

18 Ebd., S.33; Interviews mit kirgisischen Nichtregierungsorganisationen, Bischkek, März 2019.

19 Treffen des Autors mit Menschenrechtsaktivisten, Taschkent, Juni 2019; Austausch mit einem tadschikischen Wissenschaftler, 2019.

20 Siehe Erica Marat, The Politics of Police Reform: Society against the State in PostSoviet Countries, Oxford: Oxford University Press, 2018.

21 Siehe OSCE, a.a.O. (Anm. 5), S. 120.

22 Interviews mit NGO-Vertretern, Bischkek, März 2019.
23 Interview mit einem Regierungsvertreter einer westlichen Botschaft, Taschkent, Juni 2019.

24 Siehe OSCE, a.a.O. (Anm. 5), S. 145.

25 Interview mit UN-Vertretern, Taschkent, Juni 2019.

26 Henry E. Hale, Patronal Politics: Eurasian Regime Dynamics in Comparative Perspective, New York: Cambridge University Press, 2015.

27 Ebd., S. 442-445.

28 Organisation for Economic Cooperation and Development (OECD), DAC Guidelines and Reference Series: Security System Reform and Governance, 31 May 2005, S. 97, https://www.oecd-ilibrary.org /development/security-system-reform-and -governance_9789264007888-en.

29 Siehe z.B. OSZE, Beschluss Nr. 1251: Programmbüro in Duschanbe, PC. DEC/ 1251, 1. Juni 2017, https://www.osce.org/ files/f/documents/f/0/331126.pdf.

30 Siehe Mateja Steinbrück Platise, Carolyn Moser, und Anne Peters (eds.), The Legal Framework of the OSCE, Cambridge: Cambridge University Press, 2019.

31 DCAF, The Role of the Organization for Security and Co-operation in Europe (OSCE) in Security Sector Governance and Reform, 20 December 2013, S.23, https://www.dcaf.ch/sites/default/files/pu blications/documents/OSCE_SSG_Mapp ing_Study_Final_Report.pdf.

32 OSCE, a.a.O. (Anm. 8).

33 Zu Menschenrechten bei der Anti-Terrorismus-Ausbildung, siehe OSCE, a.a.O. (Anm. 5), S. 140.

34 Olimjon Bakhtaliev, „The Evolving Role of Civil Society in Peace and Security in Tajikistan: Challenges and Opportunities“, Saferworld, 17 March 2020, https://www.saferworld.org.uk/resourc es/news-and-analysis/post/861-the-evolvin g-role-of-civil-society-in-peace-and-securit y-in-tajikistan-challenges-and-opportuniti es. 
35 Bruce Cronin, „Creating Stability in the New Europe: The OSCE High Commissioner on National Minorities and the Socialization of Risky States“, in: Security Studies 12:1, 2002, 132-163.

36 Cornelius Friesendorf, How Western Soldiers Fight: Organizational Routines in Multinational Missions, Cambridge: Cambridge University Press, 2018, Kapitel 4.

37 Siehe hierzu Sarvinoz Rukhullo, „Programma reformirovaniya militsii: Voprosy bez otvetov", [Police Reform Programme: Unanswered Questions], Radio Ozodi, 23 February 2020, https:// rus.ozodi.org/a/30449643.html.

38 Karolina Kluczewska, „Benefactor, Industry or Intruder? Perceptions of Interna- tional Organizations in Central Asia: The Case of the OSCE in Tajikistan", in: Central Asian Survey 36: 3, 2017, 353-372.

39 Filippo De Danieli, „Counter-Narcotics Policies in Tajikistan and their Impact on State Building“, in: Central Asian Survey 30: 1, 2011, 129-145.

40 Bayley, a.a.O. (Anm. 2), S. 64.

41 David Lewis, „Reassessing the Role of OSCE Police Assistance Programing in Central Asia“, Occasional Paper Series No. 4, Open Society Foundations, April 2011, https:/www.opensocietyfoundatio ns.org/publications/reassessing-role-oscepolice-assistance-programing-central-asia.

42 OSCE, a.a.O. (Anm. 5), S. 124. 